$11-1-2016$

\title{
Study of the Left Censored Data from the Gumbel Type II Distribution under a Bayesian Approach
}

Tabassum Naz Sindhu

Quaid-i-Azam University, Islamabad, Pakistan, sindhuqau@gmail.com

Navid Feroze

Ripha International University, Islamabad, Pakistan, navidferoz@gmail.com

Muhammad Aslam

Ripha International University, Islamabad, Pakistan, aslamsdqu@yahoo.com

Follow this and additional works at: http://digitalcommons.wayne.edu/jmasm

Part of the Applied Statistics Commons, Social and Behavioral Sciences Commons, and the Statistical Theory Commons

\section{Recommended Citation}

Sindhu, Tabassum Naz; Feroze, Navid; and Aslam, Muhammad (2016) "Study of the Left Censored Data from the Gumbel Type II Distribution under a Bayesian Approach," Journal of Modern Applied Statistical Methods: Vol. 15 : Iss. 2 , Article 10.

DOI: $10.22237 /$ jmasm/1478002080

Available at: http://digitalcommons.wayne.edu/jmasm/vol15/iss2/10

This Regular Article is brought to you for free and open access by the Open Access Journals at DigitalCommons@WayneState. It has been accepted for inclusion in Journal of Modern Applied Statistical Methods by an authorized editor of DigitalCommons@WayneState. 


\section{Study of the Left Censored Data from the Gumbel Type II Distribution under a Bayesian Approach}

\author{
Tabassum Naz Sindhu \\ Quaid-i-Azam University \\ Islamabad, Pakistan
}

\author{
Navid Feroze \\ Ripha International University \\ Islamabad, Pakistan
}

\author{
Muhammad Aslam \\ Ripha International University \\ Islamabad, Pakistan
}

Based on left type II censored samples from a Gumbel type II distribution, the Bayes estimators and corresponding risks of the unknown parameter were obtained under different asymmetric loss functions, assuming different informative and non-informative priors. Elicitation of hyper-parameters through prior predictive approach has also been discussed. The expressions for the credible intervals and posterior predictive distributions have been derived. Comparisons of these estimators are made through simulation study using numerical and graphical methods.

Keywords: Left censoring, loss functions, credible intervals, posterior predictive distributions

\section{Introduction}

Gumbel type II distribution is very useful in life testing. Kotz and Nadarajah (2000) have given a brief characterization of the Gumbel type II distribution. Corsini, Gini, Greco, and Verrazzani (2002) studied the maximum likelihood (ML) algorithms and Cramer-Rao (CR) bounds for the location and scale parameters of the Gumbel distribution. Mousa, Jaheen, and Ahmad (2002) considered the Bayesian estimation to analyze both parameters of the Gumbel distribution based on record values.

The probability density function of the Gumbel distribution of the second kind is given by

$$
f(x)=\tau v x^{-(v+1)} \exp \left[-\tau x^{-v}\right], \quad x>0, \tau, v>0 .
$$

T. N. Sindhu is in the Department of Statistics. Email at: sindhuqau@gmail.com. N. Feroze is in the Departments of Statistics.Email at: navidferoz@hotmail.com. M. Aslam is in the Department of Statistics. Email at: aslamsdqu@yahoo.com. 


\section{SINDHU ET AL.}

The corresponding cumulative distribution function is:

$$
F(x)=1-\exp \left[-\tau x^{-v}\right], \quad x>0, \tau, v>0
$$

The parameter $v$ (being known) is a shape parameter of the model, and $\tau$ is the scale parameter.

The use of a Bayesian approach allows both sample and prior information to be incorporated into the statistical analysis, which will improve the quality of the inferences and permit a reduction in sample size. The decision-theoretic viewpoint takes into account additional information concerning the possible consequences of decisions (quantified by a loss function). The aim of this is to consider the statistical analysis of the unknown parameters when the data are left censored from the Gumbel distribution of the second kind. There is a widespread application and use of left-censoring or left-censored data in survival analysis and reliability theory. For example, in medical studies patients are subject to regular examinations. Discovery of a condition only tells us that the onset of sickness fell in the period since the previous examination and nothing about the exact date of the attack. Thus the time elapsed since onset has been left censored. Similarly, consider left-censored data when estimating functions of exact policy duration without knowing the exact date of policy entry; or when estimating functions of exact age without knowing the exact date of birth. Coburn, McBride and Ziller (2002) faced this problem due to the incidence of a higher proportion of rural children whose spells were left censored (i.e., those children who entered the sample uninsured), and who remained uninsured throughout the sample. As another example, job duration might be incomplete because the beginning of the job spells is not observed, which is an incidence of left censoring (Bagger, 2005).

\section{Likelihood Function and Posterior Distribution}

Let $X_{(r+1)}, \ldots, X_{(n)}$ be the last $n-r$ order statistics from a random sample of size $n$ following Gumbel type II distribution. Then the joint probability density function of $X_{(r+1)}, \ldots, X_{(n)}$ is given by

$$
\begin{gathered}
f\left(x_{(r+1)}, \ldots, x_{(n)} ; \tau, v\right)=\frac{n !}{r !}\left(F\left(x_{(r+1)}\right)\right)^{r} f\left(x_{(r+1)}\right) \ldots f\left(x_{(n)}\right) \\
\propto \sum_{k=0}^{r}(-1)^{k}\left(\begin{array}{l}
r \\
k
\end{array}\right) \tau^{s} \exp \left[-\tau \varsigma\left(x_{(i)}\right)\right],
\end{gathered}
$$


where $s=n-r$, and

$$
\varsigma\left(x_{(i)}\right)=\exp \left[-\tau\left\{\sum_{i=r+1}^{n} x_{(i)}^{-v}+k x_{(r+1)}^{-v}\right\}\right]
$$

\section{Prior and Posterior Distributions}

The uniform prior is assumed to be

$$
p(\tau) \propto k, \tau>0 .
$$

The posterior distribution under the uniform prior for the left censored data is:

$$
p(\tau \mid x)=\frac{\sum_{k=0}^{r}(-1)^{k}\left(\begin{array}{l}
r \\
k
\end{array}\right)(\tau)^{s} \exp \left[-\tau \varsigma\left(x_{(i)}\right)\right]}{\sum_{k=0}^{r}(-1)^{k}\left(\begin{array}{l}
r \\
k
\end{array}\right) \frac{\Gamma(s+1)}{\left\{\varsigma\left(x_{(i)}\right)\right\}^{(s+1)}}}, \tau>0 .
$$

The informative prior for the parameter $\tau$ is assumed to be exponential distribution:

$$
p(\tau)=w e^{-\tau w}, \quad w>0, \quad \tau>0 .
$$

The posterior distribution under the assumption of exponential prior is:

$$
p(\tau \mid x)=\frac{\sum_{k=0}^{r}(-1)^{k}\left(\begin{array}{l}
r \\
k
\end{array}\right)(\tau)^{s} \exp \left[-\tau\left\{w+\varsigma\left(x_{(i)}\right)\right\}\right]}{\sum_{k=0}^{r}(-1)^{k}\left(\begin{array}{l}
r \\
k
\end{array}\right) \frac{\Gamma(s+1)}{\left\{w+\varsigma\left(x_{(i)}\right)\right\}^{(s+1)}}}, \tau>0
$$

The informative prior for the parameter $\tau$ is assumed to be gamma distribution: 


\section{SINDHU ET AL.}

$$
p(\tau)=\frac{b^{a}}{\Gamma(a)} \tau^{a-1} e^{-b \tau}, \quad a, b, \tau>0 .
$$

The posterior distribution under the assumption of gamma prior for the left censored data is:

$$
p(\tau \mid x)=\frac{\sum_{k=0}^{r}(-1)^{k}\left(\begin{array}{l}
r \\
k
\end{array}\right)(\tau)^{s+a-1} \exp \left[-\tau\left\{b+\varsigma\left(x_{(i)}\right)\right\}\right]}{\sum_{k=0}^{r}(-1)^{k}\left(\begin{array}{l}
r \\
k
\end{array}\right) \frac{\Gamma(s+a)}{\left\{b+\varsigma\left(x_{(i)}\right)\right\}^{(s+a)}}}, \tau>0
$$

The informative prior for the parameter $\tau$ is assumed to be inverse Levy distribution:

$$
p(\tau)=\sqrt{\frac{c}{2 \pi}} \tau^{-\frac{1}{2}} e^{-\left(\frac{c \tau}{2}\right)}, c, \tau>0 .
$$

The posterior distribution under the inverse Levy prior for the left censored data is:

$$
p(\tau \mid x)=\frac{\sum_{k=0}^{r}(-1)^{k}\left(\begin{array}{l}
r \\
k
\end{array}\right)(\tau)^{s+\frac{1}{2}-1} \exp \left[-\tau\left\{\frac{c}{2}+\varsigma\left(x_{(i)}\right)\right\}\right]}{\sum_{k=0}^{r}(-1)^{k}\left(\begin{array}{l}
r \\
k
\end{array}\right) \frac{\Gamma\left(s+\frac{1}{2}\right)}{\left\{\frac{c}{2}+\varsigma\left(x_{(i)}\right)\right\}}}, \tau>0
$$

\section{Bayes Estimators and Posterior Risks under Different Loss Functions}

Consider the derivation of the Bayes estimator and corresponding posterior risks under different loss functions. The Bayes estimators are evaluated under precautionary loss function (PLF), weighted squared error loss function (WSELF), squared-log error loss function (SLELF), and entropy loss function (ELF). The 


\section{LEFT CENSORED DATA FROM THE GUMBEL TYPE II DISTRIBUTION}

Bayes estimator and corresponding posterior risks under different loss functions are given in the Table 1.

Table 1. Bayes estimator and posterior risks under different loss functions

\begin{tabular}{|c|c|c|c|}
\hline \multicolumn{2}{|c|}{ Loss Function $=L(\tau, \hat{\tau})$} & Bayes Estimator & Posterior Risk \\
\hline PLF: & $\frac{(\tau-\hat{\tau})^{2}}{\hat{\tau}}$ & $\sqrt{E\left(\tau^{2} \mid \mathbf{x}\right)}$ & $2\left\{\sqrt{E\left(\tau^{2} \mid x\right)}-E(\tau \mid x)\right\}$ \\
\hline WSELF: & $\frac{(\tau-\hat{\tau})^{2}}{\hat{\tau}}$ & $\left\{E\left(\tau^{-1} \mid x\right)\right\}^{-1}$ & $E(\tau \mid x)-\left\{E\left(\tau^{-1} \mid x\right)\right\}^{-1}$ \\
\hline SLELF: & $(\ln \hat{\tau}-\ln \tau)^{2}$ & $\exp \{E(\ln \tau \mid x)\}$ & $E\{(\ln \tau \mid x)\}^{2}-\{E(\ln \tau \mid x)\}^{2}$ \\
\hline ELF: & $\left\{\left(\frac{\hat{\tau}}{\tau}\right)-\ln \left(\frac{\hat{\tau}}{\tau}\right)-1\right\}$ & $\left\{E\left(\tau^{-1} \mid x\right)\right\}^{-1}$ & $\ln \left\{E\left(\tau^{-1} \mid x\right)\right\}+E(\ln \tau)$ \\
\hline
\end{tabular}

The Bayes estimators and posterior risks under uniform prior are:

$$
\begin{aligned}
& \hat{\tau}_{P L F}=\sqrt{\frac{\sum_{k=0}^{r}(-1)^{k}\left(\begin{array}{l}
r \\
k
\end{array}\right) \frac{\Gamma(s+3)}{\left\{\varsigma\left(x_{(i)}\right)\right\}^{(s+3)}}}{\sum_{k=0}^{r}(-1)^{k}\left(\begin{array}{l}
r \\
k
\end{array}\right) \frac{\Gamma(s+1)}{\left\{\varsigma\left(x_{(i)}\right)\right\}^{(s+1)}}}}, \\
& \rho\left(\hat{\tau}_{P L F}\right)=2\left[\sqrt{\frac{\sum_{k=0}^{r}(-1)^{k}\left(\begin{array}{l}
r \\
k
\end{array}\right) \frac{\Gamma(s+3)}{\left\{\varsigma\left(x_{(i)}\right)\right\}^{(s+3)}}}{\sum_{k=0}^{r}(-1)^{k}\left(\begin{array}{l}
r \\
k
\end{array}\right) \frac{\Gamma(s+1)}{\left\{\varsigma\left(x_{(i)}\right)\right\}^{(s+1)}}}}-\frac{\sum_{k=0}^{r}(-1)^{k}\left(\begin{array}{l}
r \\
k
\end{array}\right) \frac{\Gamma(s+2)}{\left\{\varsigma\left(x_{(i)}\right)\right\}^{(s+2)}}}{\sum_{k=0}^{r}(-1)^{k}\left(\begin{array}{l}
r \\
k
\end{array}\right) \frac{\Gamma(s+1)}{\left\{\varsigma\left(x_{(i)}\right)\right\}^{(s+1)}}}\right]
\end{aligned}
$$




\section{SINDHU ET AL.}

$$
\begin{aligned}
& \hat{\tau}_{\text {WSELF }}=\frac{\sum_{k=0}^{r}(-1)^{k}\left(\begin{array}{l}
r \\
k
\end{array}\right) \frac{\Gamma(s+1)}{\left\{\varsigma\left(x_{(i)}\right)\right\}^{(s+1)}}}{\sum_{k=0}^{r}(-1)^{k}\left(\begin{array}{l}
r \\
k
\end{array}\right) \frac{\Gamma(s)}{\left\{\varsigma\left(x_{(i)}\right)\right\}^{(s)}}}, \\
& \rho\left(\hat{\tau}_{\text {WSELF }}\right)=\left[\frac{\sum_{k=0}^{r}(-1)^{k}\left(\begin{array}{l}
r \\
k
\end{array}\right) \frac{\Gamma(s+2)}{\left\{\varsigma\left(x_{(i)}\right)\right\}^{(s+2)}}}{\sum_{k=0}^{r}(-1)^{k}\left(\begin{array}{l}
r \\
k
\end{array}\right) \frac{\Gamma(s+1)}{\left\{\varsigma\left(x_{(i)}\right)\right\}^{(s+1)}}}-\frac{\sum_{k=0}^{r}(-1)^{k}\left(\begin{array}{l}
r \\
k
\end{array}\right) \frac{\Gamma(s+1)}{\left\{\varsigma\left(x_{(i)}\right)\right\}^{(s+1)}}}{\sum_{k=0}^{r}(-1)^{k}\left(\begin{array}{l}
r \\
k
\end{array}\right) \frac{\Gamma(s)}{\left\{\varsigma\left(x_{(i)}\right)\right\}^{(s)}}}\right] . \\
& \hat{\tau}_{\text {SLELF }}=\frac{\sum_{k=0}^{r}(-1)^{k}\left(\begin{array}{l}
r \\
k
\end{array}\right) \frac{\Gamma(s+1) \exp \{\psi(s+1)\}}{\left\{\varsigma\left(x_{(i)}\right)\right\}^{(s+2)}}}{\sum_{k=0}^{r}(-1)^{k}\left(\begin{array}{l}
r \\
k
\end{array}\right) \frac{\Gamma(s+1)}{\left\{\varsigma\left(x_{(i)}\right)\right\}^{(s+1)}}}, \\
& \rho\left(\hat{\tau}_{\text {SLELF }}\right)=\frac{\sum_{k=0}^{r}(-1)^{k}\left(\begin{array}{l}
r \\
k
\end{array}\right) \frac{\Gamma(s+1) \psi^{\prime}(s+1)}{\left\{\varsigma\left(x_{(i)}\right)\right\}^{(s+1)}}}{\sum_{k=0}^{r}(-1)^{k}\left(\begin{array}{l}
r \\
k
\end{array}\right) \frac{\Gamma(s+1)}{\left\{\varsigma\left(x_{(i)}\right)\right\}^{(s+1)}}} . \\
& \hat{\tau}_{E L F}=\frac{\sum_{k=0}^{r}(-1)^{k}\left(\begin{array}{l}
r \\
k
\end{array}\right) \frac{\Gamma(s+1)}{\left\{\varsigma\left(x_{(i)}\right)\right\}^{(s+1)}}}{\sum_{k=0}^{r}(-1)^{k}\left(\begin{array}{l}
r \\
k
\end{array}\right) \frac{\Gamma(s)}{\left\{\varsigma\left(x_{(i)}\right)\right\}^{(s)}}},
\end{aligned}
$$




$$
\rho\left(\hat{\tau}_{E L F}\right)=\frac{\sum_{k=0}^{r}(-1)^{k}\left(\begin{array}{l}
r \\
k
\end{array}\right) \frac{\Gamma(s)}{\left\{\varsigma\left(x_{(i)}\right)\right\}^{(s)}}}{\sum_{k=0}^{r}(-1)^{k}\left(\begin{array}{l}
r \\
k
\end{array}\right) \frac{\Gamma(s+1)}{\left\{\varsigma\left(x_{(i)}\right)\right\}^{(s+1)}}} \psi(s+1)-\ln \left[\frac{\sum_{k=0}^{r}(-1)^{k}\left(\begin{array}{l}
r \\
k
\end{array}\right) \frac{1}{\left\{\varsigma\left(x_{(i)}\right)\right\}^{(s+1)}}}{\sum_{k=0}^{r}(-1)^{k}\left(\begin{array}{l}
r \\
k
\end{array}\right) \frac{1}{\left\{\varsigma\left(x_{(i)}\right)\right\}^{(s+2)}}}\right] .
$$

The Bayes estimators and posterior risks under the rest of priors can be obtained in a similar manner.

\section{Bayes Credible Interval for the Left Censored Data}

The Bayesian credible intervals for type II left censored data under informative and non-informative priors, as discussed by Saleem and Aslam (2009) are presented in the following. The credible intervals for type II left censored data under all priors are:

$$
\begin{aligned}
& \frac{\chi_{2(s+1)\left(\frac{\alpha}{2}\right)}^{2} \sum_{k=0}^{r}(-1)^{k}\left(\begin{array}{l}
r \\
k
\end{array}\right) \frac{1}{\left\{\varsigma\left(x_{(i)}\right)\right\}^{(s+2)}}}{2 \sum_{k=0}^{r}(-1)^{k}\left(\begin{array}{l}
r \\
k
\end{array}\right) \frac{1}{\left\{\varsigma\left(x_{(i)}\right)\right\}^{(s+1)}}}<\tau_{\text {Uniform }}<\frac{\chi_{2(s+1)\left(1-\frac{\alpha}{2}\right)}^{2} \sum_{k=0}^{r}(-1)^{k}\left(\begin{array}{l}
r \\
k
\end{array}\right) \frac{1}{\left\{\varsigma\left(x_{(i)}\right)\right\}^{(s+2)}}}{2 \sum_{k=0}^{r}(-1)^{k}\left(\begin{array}{l}
r \\
k
\end{array}\right) \frac{1}{\left\{\varsigma\left(x_{(i)}\right)\right\}^{(s+1)}}} \\
& \frac{\chi_{2(s+1)\left(\frac{\alpha}{2}\right)}^{2} \sum_{k=0}^{r}(-1)^{k}\left(\begin{array}{l}
r \\
k
\end{array}\right) \frac{1}{\left\{w+\varsigma\left(x_{(i)}\right)\right\}^{(s+2)}}}{2 \sum_{k=0}^{r}(-1)^{k}\left(\begin{array}{l}
r \\
k
\end{array}\right) \frac{1}{\left\{w+\varsigma\left(x_{(i)}\right)\right\}^{(s+1)}}}<\tau_{\text {Exponential }}<\frac{\chi_{2(s+1)\left(1-\frac{\alpha}{2}\right)}^{2} \sum_{k=0}^{r}(-1)^{k}\left(\begin{array}{l}
r \\
k
\end{array}\right) \frac{1}{\left\{w+\varsigma\left(x_{(i)}\right)\right\}^{(s+2)}}}{2 \sum_{k=0}^{r}(-1)^{k}\left(\begin{array}{l}
r \\
k
\end{array}\right) \frac{1}{\left\{w+\varsigma\left(x_{(i)}\right)\right\}^{(s+1)}}} \\
& \frac{\chi_{2(s+1)\left(\frac{\alpha}{2}\right)}^{2} \sum_{k=0}^{r}(-1)^{k}\left(\begin{array}{l}
r \\
k
\end{array}\right) \frac{1}{\left\{b+\varsigma\left(x_{(i)}\right)\right\}^{(s+a+1)}}}{\left\{\sum_{k=0}^{r}(-1)^{k}\left(\begin{array}{l}
r \\
k
\end{array}\right) \frac{1}{\left\{b+\varsigma\left(x_{(i)}\right)\right\}^{(s+a)}}\right.}<\frac{\chi_{2(s+1)\left(1-\frac{\alpha}{2}\right)}^{2} \sum_{k=0}^{r}(-1)^{k}\left(\begin{array}{l}
r \\
k
\end{array}\right) \frac{1}{\left\{b+\varsigma\left(x_{(i)}\right)\right\}^{(s+a+1)}}}{2 \sum_{k=0}^{r}(-1)^{k}\left(\begin{array}{l}
r \\
k
\end{array}\right) \frac{1}{\left\{b+\varsigma\left(x_{(i)}\right)\right\}^{(s+a)}}}
\end{aligned}
$$




\section{SINDHU ET AL.}

$$
\frac{\chi_{2(s+1)\left(\frac{\alpha}{2}\right)}^{2} \sum_{k=0}^{r}(-1)^{k}\left(\begin{array}{l}
r \\
k
\end{array}\right) \frac{1}{\left\{c / 2+\varsigma\left(x_{(i)}\right)\right\}^{\left(s+\frac{3}{2}\right)}}}{2 \sum_{k=0}^{r}(-1)^{k}\left(\begin{array}{l}
r \\
k
\end{array}\right) \frac{1}{\left\{c / 2+\varsigma\left(x_{(i)}\right)\right\}^{(s+1 / 2)}}}<\tau_{I n-\text { Levy }}<\frac{\chi_{2(s+1)\left(1-\frac{\alpha}{2}\right)}^{2} \sum_{k=0}^{r}(-1)^{k}\left(\begin{array}{l}
r \\
k
\end{array}\right) \frac{1}{\left\{c / 2+\varsigma\left(x_{(i)}\right)\right\}^{(s+3 / 2)}}}{2 \sum_{k=0}^{r}(-1)^{k}\left(\begin{array}{l}
r \\
k
\end{array}\right) \frac{1}{\left\{c / 2+\varsigma\left(x_{(i)}\right)\right\}^{(s+1 / 2)}}}
$$

\section{Elicitation}

Consider a probability elicitation method known as prior predictive elicitation. Predictive elicitation is a method for estimating hyper-parameters of prior distributions by inverting corresponding prior predictive distributions. Elicitation of hyper-parameter from the prior $p(\tau)$ is conceptually difficult task because we first have to identify prior distribution and then its hyper-parameters. The prior predictive distribution is used for the elicitation of the hyper-parameters which is compared with the experts' judgment about this distribution and then the hyperparameters are chosen in such a way so as to make the judgment agree closely as possible with the given distribution (see Grimshaw, 1993; Kadane, 1980; O'Hagan et al., 2006; Grimshaw, Collings, Larsen, \& Hurt, 2001; Jenkinson, 2005; and León, Vázquez-Polo, \& González, 2003).

According to Aslam (2003), the method of assessment is to compare the predictive distribution with experts' assessment about this distribution and then to choose the hyper-parameters that make the assessment agree closely with the member of the family. He discusses three important methods to elicit the hyperparameters: (i) via the prior predictive probabilities (ii) via elicitation of the confidence levels (iii) via the predictive mode and confidence level. We will use the prior predictive approach by Aslam (2003).

\section{Prior predictive distribution}

The prior predictive distribution is:

$$
p(y)=\int_{0}^{\infty} p(y \mid \tau) p(\tau) d \tau
$$

The predictive distribution under exponential prior is: 


$$
p(y)=\int_{0}^{\infty} w v \tau y^{-(v+1)} \exp \left\{-\tau\left(y^{-v}+w\right)\right\} d \tau
$$

After some simplification it reduces as

$$
p(y)=\frac{v w}{y^{(v+1)}\left\{w+y^{-v}\right\}^{2}}, \quad y>0 .
$$

The predictive distribution under gamma prior is:

$$
\begin{gathered}
p(y)=\frac{v a b^{a}}{y^{(v+1)}\left\{b+y^{-v}\right\}^{a+1}}, \quad 0<y<\infty . \\
p(y)=\frac{v \sqrt{c}}{2^{3 / 2} y^{(v+1)}\left\{c / 2+y^{-v}\right\}^{3 / 2}}, \quad 0<y<\infty .
\end{gathered}
$$

By using the method of elicitation defined by Aslam (2003), we obtain the

following hyper-parameters $w=0.798566, a=0.152109, \quad b=6.523695$ and $c=15.985795$.

\section{Posterior Predictive Distribution}

The predictive distribution contains the information about the independent future random observation given preceding observations. The reader desire more details can see Bansal (2007).

The posterior predictive distribution of the future observation $y=x_{n+1}$ is

$$
p(y \mid \mathbf{x})=\int_{0}^{\infty} p(\tau \mid \mathbf{x}) p(y \mid \tau) d \tau
$$




\section{SINDHU ET AL.}

Where $p(y)=\tau v x^{-(v+1)} \exp \left[-\tau x^{-v}\right]$, is the future observation density and $p(\tau \mid \mathbf{x})$ is the posterior distribution obtained by incorporating the likelihood with the respective prior distributions.

The posterior predictive distribution of the future observation $y=x_{n+1}$ under uniform prior is

$$
p(y \mid x)=\frac{\sum_{k=0}^{r}(-1)^{k}\left(\begin{array}{l}
r \\
k
\end{array}\right) \frac{s+1}{y^{(v+1)}\left\{\varsigma\left(x_{(i)}\right)+y^{-v}\right\}^{(s+2)}}}{\sum_{k=0}^{r}(-1)^{k}\left(\begin{array}{l}
r \\
k
\end{array}\right) \frac{1}{\left\{\varsigma\left(x_{(i)}\right)\right\}^{(s+1)}}}, y>0 .
$$

The posterior predictive distribution of the future observation $y=x_{n+1}$ under exponential prior is

$$
p(y \mid x)=\frac{\sum_{k=0}^{r}(-1)^{k}\left(\begin{array}{l}
r \\
k
\end{array}\right) \frac{s+1}{y^{(v+1)}\left\{w+\varsigma\left(x_{(i)}\right)+y^{-v}\right\}^{(s+2)}}}{\sum_{k=0}^{r}(-1)^{k}\left(\begin{array}{l}
r \\
k
\end{array}\right) \frac{1}{\left\{w+\varsigma\left(x_{(i)}\right)\right\}^{(s+1)}}}, y>0 .
$$

The posterior predictive distribution of the future observation $y=x_{n+1}$ under gamma prior is

$$
p(y \mid x)=\frac{\sum_{k=0}^{r}(-1)^{k}\left(\begin{array}{l}
r \\
k
\end{array}\right) \frac{s+a}{y^{(v+1)}\left\{b+\varsigma\left(x_{(i)}\right)+y^{-v}\right\}^{(s+a+1)}}}{\sum_{k=0}^{r}(-1)^{k}\left(\begin{array}{l}
r \\
k
\end{array}\right) \frac{1}{\left\{b+\varsigma\left(x_{(i)}\right)\right\}^{(s+a)}}}, y>0 .
$$

The posterior predictive distribution of the future observation $y=x_{n+1}$ under Inverse-Levy prior is 


$$
p(y \mid x)=\frac{\sum_{k=0}^{r}(-1)^{k}\left(\begin{array}{l}
r \\
k
\end{array}\right) \frac{s+1 / 2}{y^{(v+1)}\left\{c / 2+\varsigma\left(x_{(i)}\right)+y^{-\nu}\right\}^{(s+3 / 2)}}}{\sum_{k=0}^{r}(-1)^{k}\left(\begin{array}{l}
r \\
k
\end{array}\right) \frac{1}{\left\{c / 2+\varsigma\left(x_{(i)}\right)\right\}^{(s+1 / 2)}}}, y>0 .
$$

\section{Simulation Study}

Simulations can be helpful and an illuminating way to approach problems in Bayesian analysis. Bayesian problems of updating estimates can be handled easily and straight forwardly with simulation. Because the distribution function of the Gumbel type II distribution can be expressed, as well as its inverse in closed form, the inversion method of simulation is straightforward to implement. The study was carried out for different values of $(n, r)$ using $\tau \in 2.5$ and $v=0.5$. Censoring rates are assumed to be $5 \%$ and $10 \%$.

Sample size is varied to observe the effect of small and large samples on the estimators. Changes in the estimators and their risks have been determined when changing the loss function and the prior distribution of $\tau$ while keeping the sample size fixed. All these results are based on 5,000 repetitions. Tables 2-6 give the estimated value of the parameter, posterior risks and 95\% confidence limits (Lower Confidence Limit (LCL) and Upper Confidence Limit (UCL)) for the parameter. The results are summarized in the following Tables and Figures 1-8. The amounts of posterior risks have been presented in the parenthesis in the tables. 


\section{SINDHU ET AL.}

Table 2. Bayes estimates and the posterior risks under PLF for $\tau \in 2.5$.

\begin{tabular}{|c|c|c|c|}
\hline \multirow{2}{*}{$\mathrm{n}$} & \multicolumn{3}{|c|}{ Uniform Prior } \\
\hline & No Censoring & $5 \%$ Censoring & $10 \%$ Censoring \\
\hline 20 & $\begin{array}{r}2.737920 \\
(0.125898)\end{array}$ & $\begin{array}{r}3.35045 \\
(0.157935)\end{array}$ & $\begin{array}{r}3.77639 \\
(0.181710)\end{array}$ \\
\hline 40 & $\begin{array}{r}2.677940 \\
(0.064145)\end{array}$ & $\begin{array}{r}3.15159 \\
(0.077609)\end{array}$ & $\begin{array}{r}3.64915 \\
(0.097539)\end{array}$ \\
\hline 60 & $\begin{array}{r}2.62145 \\
(0.042453)\end{array}$ & $\begin{array}{r}3.09163 \\
(0.051534)\end{array}$ & $\begin{array}{r}3.54489 \\
(0.060447)\end{array}$ \\
\hline 80 & $\begin{array}{r}2.57594 \\
(0.031510)\end{array}$ & $\begin{array}{r}3.04116 \\
(0.038311)\end{array}$ & $\begin{array}{r}3.50579 \\
(0.045182)\end{array}$ \\
\hline 100 & $\begin{array}{r}2.56138 \\
(0.025173)\end{array}$ & $\begin{array}{r}3.03806 \\
(0.030759)\end{array}$ & $\begin{array}{r}3.47670 \\
(0.036015)\end{array}$ \\
\hline $\mathrm{n}$ & \multicolumn{3}{|c|}{ Exponential Prior } \\
\hline 20 & $\begin{array}{r}2.58014 \\
(0.118643)\end{array}$ & $\begin{array}{r}2.96201 \\
(0.138226)\end{array}$ & $\begin{array}{r}3.38135 \\
(0.156758)\end{array}$ \\
\hline 40 & $\begin{array}{r}2.52198 \\
(0.060409)\end{array}$ & $\begin{array}{r}2.95898 \\
(0.072220)\end{array}$ & $\begin{array}{r}3.36035 \\
(0.084258)\end{array}$ \\
\hline 60 & $\begin{array}{r}2.52440 \\
(0.040720)\end{array}$ & $\begin{array}{r}2.95009 \\
(0.049112)\end{array}$ & $\begin{array}{r}3.35418 \\
(0.057015)\end{array}$ \\
\hline 80 & $\begin{array}{r}2.52171 \\
(0.030847)\end{array}$ & $\begin{array}{r}2.94949 \\
(0.037501)\end{array}$ & $\begin{array}{r}3.33655 \\
(0.043241)\end{array}$ \\
\hline 100 & $\begin{array}{r}2.50779 \\
(0.024647) \\
\end{array}$ & $\begin{array}{r}2.92773 \\
(0.030070) \\
\end{array}$ & $\begin{array}{r}3.30688 \\
(0.035032) \\
\end{array}$ \\
\hline $\mathrm{n}$ & \multicolumn{3}{|c|}{ Gamma Prior } \\
\hline 20 & $\begin{array}{r}1.43895 \\
(0.068852)\end{array}$ & $\begin{array}{r}1.55700 \\
(0.075152)\end{array}$ & $\begin{array}{r}1.64308 \\
(0.079688)\end{array}$ \\
\hline 40 & $\begin{array}{r}1.82853 \\
(0.044707)\end{array}$ & $\begin{array}{r}2.04504 \\
(0.050801)\end{array}$ & $\begin{array}{r}2.21285 \\
(0.055460)\end{array}$ \\
\hline 60 & $\begin{array}{r}2.00816 \\
(0.032974)\end{array}$ & $\begin{array}{r}2.26658 \\
(0.037962)\end{array}$ & $\begin{array}{r}2.49874 \\
(0.042352)\end{array}$ \\
\hline 80 & $\begin{array}{r}2.11237 \\
(0.026111)\end{array}$ & $\begin{array}{r}2.41150 \\
(0.030475)\end{array}$ & $\begin{array}{r}2.67252 \\
(0.034264)\end{array}$ \\
\hline 100 & $\begin{array}{r}2.218482 \\
(0.021653)\end{array}$ & $\begin{array}{r}2.51014 \\
(0.025478) \\
\end{array}$ & $\begin{array}{r}2.79600 \\
(0.028819) \\
\end{array}$ \\
\hline $\mathrm{n}$ & \multicolumn{3}{|c|}{ Inverse Levy Prior } \\
\hline 20 & $\begin{array}{r}1.32737 \\
(0.062473)\end{array}$ & $\begin{array}{r}1.43304 \\
(0.067927)\end{array}$ & $\begin{array}{r}1.49803 \\
(0.071294)\end{array}$ \\
\hline 40 & $\begin{array}{r}1.72182 \\
(0.041743)\end{array}$ & $\begin{array}{r}1.91963 \\
(0.047193)\end{array}$ & $\begin{array}{r}2.05833 \\
(0.051005)\end{array}$ \\
\hline 60 & $\begin{array}{r}1.93203 \\
(0.031544)\end{array}$ & $\begin{array}{r}2.16662 \\
(0.036031)\end{array}$ & $\begin{array}{r}2.37030 \\
(0.039845)\end{array}$ \\
\hline 80 & $\begin{array}{r}2.04177 \\
(0.025129)\end{array}$ & $\begin{array}{r}2.32593 \\
(0.029234)\end{array}$ & $\begin{array}{r}2.55092 \\
(0.032477)\end{array}$ \\
\hline 100 & $\begin{array}{r}2.12131 \\
(0.020951)\end{array}$ & $\begin{array}{r}2.41626 \\
(0.024413)\end{array}$ & $\begin{array}{r}2.68807 \\
(0.027552)\end{array}$ \\
\hline
\end{tabular}




\section{LEFT CENSORED DATA FROM THE GUMBEL TYPE II DISTRIBUTION}

Table 3. Bayes estimates and the posterior risks under WSELF for $\tau \in 2.5$.

\begin{tabular}{|c|c|c|c|}
\hline \multirow{2}{*}{$\mathrm{n}$} & \multicolumn{3}{|c|}{ Uniform Prior } \\
\hline & No Censoring & $5 \%$ Censoring & $10 \%$ Censoring \\
\hline 20 & $\begin{array}{r}2.66809 \\
(0.133405)\end{array}$ & $\begin{array}{r}3.08160 \\
(0.157976)\end{array}$ & $\begin{array}{r}3.54947 \\
(0.186003)\end{array}$ \\
\hline 40 & $\begin{array}{r}2.55583 \\
(0.063896)\end{array}$ & $\begin{array}{r}3.05530 \\
(0.078578)\end{array}$ & $\begin{array}{r}3.43934 \\
(0.090409)\end{array}$ \\
\hline 60 & $\begin{array}{r}2.55213 \\
(0.042536)\end{array}$ & $\begin{array}{r}3.02388 \\
(0.051901)\end{array}$ & $\begin{array}{r}3.42741 \\
(0.060168)\end{array}$ \\
\hline 80 & $\begin{array}{r}2.53489 \\
(0.031686)\end{array}$ & $\begin{array}{r}3.01692 \\
(0.038842)\end{array}$ & $\begin{array}{r}3.41996 \\
(0.04506)\end{array}$ \\
\hline 100 & $\begin{array}{r}2.51670 \\
(0.025167)\end{array}$ & $\begin{array}{r}3.00774 \\
(0.030991)\end{array}$ & $\begin{array}{r}3.40597 \\
(0.035925)\end{array}$ \\
\hline $\mathrm{n}$ & \multicolumn{3}{|c|}{ Exponential Prior } \\
\hline 20 & $\begin{array}{r}2.37956 \\
(0.118978)\end{array}$ & $\begin{array}{r}2.93114 \\
(0.139567)\end{array}$ & $\begin{array}{r}3.35007 \\
(0.158471)\end{array}$ \\
\hline 40 & $\begin{array}{r}2.42840 \\
(0.060710)\end{array}$ & $\begin{array}{r}2.87664 \\
(0.073818)\end{array}$ & $\begin{array}{r}3.27245 \\
(0.085679)\end{array}$ \\
\hline 60 & $\begin{array}{r}2.46768 \\
(0.041128)\end{array}$ & $\begin{array}{r}2.85571 \\
(0.049693)\end{array}$ & $\begin{array}{r}3.270610 \\
(0.057314)\end{array}$ \\
\hline 80 & $\begin{array}{r}2.47487 \\
(0.030936)\end{array}$ & $\begin{array}{r}2.72288 \\
(0.037589)\end{array}$ & $\begin{array}{r}3.134120 \\
(0.043824)\end{array}$ \\
\hline 100 & $\begin{array}{r}2.48550 \\
(0.024855)\end{array}$ & $\begin{array}{r}2.624320 \\
(0.030108)\end{array}$ & $\begin{array}{r}3.02926 \\
(0.035046)\end{array}$ \\
\hline $\mathrm{n}$ & \multicolumn{3}{|c|}{ Gamma Prior } \\
\hline 20 & $\begin{array}{r}1.33348 \\
(0.069626)\end{array}$ & $\begin{array}{r}1.44368 \\
(0.075839)\end{array}$ & $\begin{array}{r}1.51586 \\
(0.080755)\end{array}$ \\
\hline 40 & $\begin{array}{r}1.75474 \\
(0.044819)\end{array}$ & $\begin{array}{r}1.98012 \\
(0.050968)\end{array}$ & $\begin{array}{r}2.12591 \\
(0.055810)\end{array}$ \\
\hline 60 & $\begin{array}{r}1.95524 \\
(0.03306)\end{array}$ & $\begin{array}{r}2.25507 \\
(0.038435)\end{array}$ & $\begin{array}{r}2.44299 \\
(0.042656)\end{array}$ \\
\hline 80 & $\begin{array}{r}2.07625 \\
(0.026231)\end{array}$ & $\begin{array}{r}2.40362 \\
(0.030624)\end{array}$ & $\begin{array}{r}2.63342 \\
(0.034421)\end{array}$ \\
\hline 100 & $\begin{array}{r}2.244640 \\
(0.021630)\end{array}$ & $\begin{array}{r}2.50664 \\
(0.025501) \\
\end{array}$ & $\begin{array}{r}2.77998 \\
(0.029085)\end{array}$ \\
\hline $\mathrm{n}$ & \multicolumn{3}{|c|}{ Inverse Levy Prior } \\
\hline 20 & $\begin{array}{r}1.24650 \\
(0.063923)\end{array}$ & $\begin{array}{r}1.31807 \\
(0.068090)\end{array}$ & $\begin{array}{r}1.38627 \\
(0.071871)\end{array}$ \\
\hline 40 & $\begin{array}{r}1.665110 \\
(0.042155)\end{array}$ & $\begin{array}{r}1.74892 \\
(0.044659)\end{array}$ & $\begin{array}{r}1.84547 \\
(0.047385)\end{array}$ \\
\hline 60 & $\begin{array}{r}1.86831 \\
(0.031400)\end{array}$ & $\begin{array}{r}2.10212 \\
(0.035987)\end{array}$ & $\begin{array}{r}2.32167 \\
(0.040176)\end{array}$ \\
\hline 80 & $\begin{array}{r}1.99783 \\
(0.02513)\end{array}$ & $\begin{array}{r}2.33427 \\
(0.030086)\end{array}$ & $\begin{array}{r}2.50929 \\
(0.032640)\end{array}$ \\
\hline 100 & $\begin{array}{r}2.18089 \\
(0.020913)\end{array}$ & $\begin{array}{r}2.40249 \\
(0.024701)\end{array}$ & $\begin{array}{r}2.64028 \\
(0.027546)\end{array}$ \\
\hline
\end{tabular}




\section{SINDHU ET AL.}

Table 4. Bayes estimates and the posterior risks under SLELF for $\tau \in 2.5$.

\begin{tabular}{|c|c|c|c|}
\hline \multirow{2}{*}{$\mathrm{n}$} & \multicolumn{3}{|c|}{ Uniform Prior } \\
\hline & No Censoring & $5 \%$ Censoring & $10 \%$ Censoring \\
\hline 20 & $\begin{array}{r}2.70493 \\
(0.048771)\end{array}$ & $\begin{array}{r}3.16249 \\
(0.051271)\end{array}$ & $\begin{array}{r}3.67867 \\
(0.054041)\end{array}$ \\
\hline 40 & $\begin{array}{r}2.60860 \\
(0.024690)\end{array}$ & $\begin{array}{r}3.08320 \\
(0.025973)\end{array}$ & $\begin{array}{r}3.52510 \\
(0.027396)\end{array}$ \\
\hline 60 & $\begin{array}{r}2.548760 \\
(0.016529)\end{array}$ & $\begin{array}{r}3.04864 \\
(0.017391)\end{array}$ & $\begin{array}{r}3.48125 \\
(0.018348)\end{array}$ \\
\hline 80 & $\begin{array}{r}2.53947 \\
(0.012422)\end{array}$ & $\begin{array}{r}3.02895 \\
(0.013072)\end{array}$ & $\begin{array}{r}3.46749 \\
(0.013793)\end{array}$ \\
\hline 100 & $\begin{array}{r}2.53070 \\
(0.009950)\end{array}$ & $\begin{array}{r}3.019810 \\
(0.010471)\end{array}$ & $\begin{array}{r}3.24692 \\
(0.011050)\end{array}$ \\
\hline $\mathrm{n}$ & \multicolumn{3}{|c|}{ Exponential Prior } \\
\hline 20 & $\begin{array}{r}2.42262 \\
(0.048771)\end{array}$ & $\begin{array}{r}2.89396 \\
(0.051271)\end{array}$ & $\begin{array}{r}3.13621 \\
(0.054041)\end{array}$ \\
\hline 40 & $\begin{array}{r}2.46614 \\
(0.024690)\end{array}$ & $\begin{array}{r}2.87997 \\
(0.025973)\end{array}$ & $\begin{array}{r}3.11318 \\
(0.027396)\end{array}$ \\
\hline 60 & $\begin{array}{r}2.47732 \\
(0.016529)\end{array}$ & $\begin{array}{r}2.79474 \\
(0.017391)\end{array}$ & $\begin{array}{r}3.01411 \\
(0.018348)\end{array}$ \\
\hline 80 & $\begin{array}{r}2.48808 \\
(0.012422)\end{array}$ & $\begin{array}{r}2.64583 \\
(0.013072)\end{array}$ & $\begin{array}{r}3.006108 \\
(0.013793)\end{array}$ \\
\hline 100 & $\begin{array}{r}2.497560 \\
(0.009950)\end{array}$ & $\begin{array}{r}2.60852 \\
(0.010471) \\
\end{array}$ & $\begin{array}{r}2.985631 \\
(0.011050)\end{array}$ \\
\hline $\mathrm{n}$ & \multicolumn{3}{|c|}{ Gamma Prior } \\
\hline 20 & $\begin{array}{r}1.37081 \\
(0.050874)\end{array}$ & $\begin{array}{r}1.48503 \\
(0.0536004)\end{array}$ & $\begin{array}{r}1.56354 \\
(0.056635)\end{array}$ \\
\hline 40 & $\begin{array}{r}1.78940 \\
(0.025218)\end{array}$ & $\begin{array}{r}1.98832 \\
(0.026557)\end{array}$ & $\begin{array}{r}2.15504 \\
(0.028047)\end{array}$ \\
\hline 60 & $\begin{array}{r}1.98230 \\
(0.016764)\end{array}$ & $\begin{array}{r}2.23221 \\
(0.017651)\end{array}$ & $\begin{array}{r}2.45581 \\
(0.018638)\end{array}$ \\
\hline 80 & $\begin{array}{r}2.081680 \\
(0.012554)\end{array}$ & $\begin{array}{r}2.38376 \\
(0.013218)\end{array}$ & $\begin{array}{r}2.63859 \\
(0.013956)\end{array}$ \\
\hline 100 & $\begin{array}{r}2.26264 \\
(0.010035) \\
\end{array}$ & $\begin{array}{r}2.48866 \\
(0.010565) \\
\end{array}$ & $\begin{array}{r}2.77011 \\
(0.011154) \\
\end{array}$ \\
\hline $\mathrm{n}$ & \multicolumn{3}{|c|}{ Inverse Levy Prior } \\
\hline 20 & $\begin{array}{r}1.27054 \\
(0.049989)\end{array}$ & $\begin{array}{r}1.34243 \\
(0.052619)\end{array}$ & $\begin{array}{r}1.42286 \\
(0.055541)\end{array}$ \\
\hline 40 & $\begin{array}{r}1.69351 \\
(0.024999)\end{array}$ & $\begin{array}{r}1.86554 \\
(0.026314)\end{array}$ & $\begin{array}{r}2.01136 \\
(0.027776)\end{array}$ \\
\hline 60 & $\begin{array}{r}1.90254 \\
(0.016663)\end{array}$ & $\begin{array}{r}2.19742 \\
(0.017856)\end{array}$ & $\begin{array}{r}2.32432 \\
(0.018518)\end{array}$ \\
\hline 80 & $\begin{array}{r}2.01472 \\
(0.012499)\end{array}$ & $\begin{array}{r}2.29894 \\
(0.013158)\end{array}$ & $\begin{array}{r}2.52262 \\
(0.013889)\end{array}$ \\
\hline 100 & $\begin{array}{r}2.20627 \\
(0.009999)\end{array}$ & $\begin{array}{r}2.40058 \\
(0.010526)\end{array}$ & $\begin{array}{r}2.64965 \\
(0.011111)\end{array}$ \\
\hline
\end{tabular}




\section{LEFT CENSORED DATA FROM THE GUMBEL TYPE II DISTRIBUTION}

Table 5. Bayes estimates and the posterior risks under ELF for $\tau \in 2.5$.

\begin{tabular}{|c|c|c|c|}
\hline \multirow{2}{*}{$\mathrm{n}$} & \multicolumn{3}{|c|}{ Uniform Prior } \\
\hline & No Censoring & $5 \%$ Censoring & $10 \%$ Censoring \\
\hline 20 & $\begin{array}{r}2.63866 \\
(0.024792)\end{array}$ & $\begin{array}{r}3.10757 \\
(0.025787)\end{array}$ & $\begin{array}{r}3.56083 \\
(0.026520)\end{array}$ \\
\hline 40 & $\begin{array}{r}2.56586 \\
(0.012448)\end{array}$ & $\begin{array}{r}3.06196 \\
(0.012508)\end{array}$ & $\begin{array}{r}3.46458 \\
(0.012576)\end{array}$ \\
\hline 60 & $\begin{array}{r}2.53490 \\
(0.008310)\end{array}$ & $\begin{array}{r}3.03388 \\
(0.008570)\end{array}$ & $\begin{array}{r}3.42366 \\
(0.008987)\end{array}$ \\
\hline 80 & $\begin{array}{r}2.52287 \\
(0.006237)\end{array}$ & $\begin{array}{r}3.00312 \\
(0.006286)\end{array}$ & $\begin{array}{r}3.15751 \\
(0.006721)\end{array}$ \\
\hline 100 & $\begin{array}{r}2.51440 \\
(0.004992)\end{array}$ & $\begin{array}{r}2.901795 \\
(0.005235)\end{array}$ & $\begin{array}{r}3.003575 \\
(0.005982)\end{array}$ \\
\hline $\mathrm{n}$ & \multicolumn{3}{|c|}{ Exponential Prior } \\
\hline 20 & $\begin{array}{r}2.56510 \\
(0.024792)\end{array}$ & $\begin{array}{r}2.69689 \\
(0.025787)\end{array}$ & $\begin{array}{r}3.05465 \\
(0.026520)\end{array}$ \\
\hline 40 & $\begin{array}{r}2.52434 \\
(0.012448)\end{array}$ & $\begin{array}{r}2.58528 \\
(0.012508)\end{array}$ & $\begin{array}{r}3.02735 \\
(0.012576)\end{array}$ \\
\hline 60 & $\begin{array}{r}2.50708 \\
(0.008310)\end{array}$ & $\begin{array}{r}2.561238 \\
(0.008570)\end{array}$ & $\begin{array}{r}3.017921 \\
(0.008987)\end{array}$ \\
\hline 80 & $\begin{array}{r}2.48248 \\
(0.006237)\end{array}$ & $\begin{array}{r}2.52515 \\
(0.006286)\end{array}$ & $\begin{array}{r}3.00984 \\
(0.006721)\end{array}$ \\
\hline 100 & $\begin{array}{r}2.46838 \\
(0.004992)\end{array}$ & $\begin{array}{r}2.49894 \\
(0.005235)\end{array}$ & $\begin{array}{r}2.91496 \\
(0.005982)\end{array}$ \\
\hline $\mathrm{n}$ & \multicolumn{3}{|c|}{ Gamma Prior } \\
\hline 20 & $\begin{array}{r}1.33972 \\
(0.025879)\end{array}$ & $\begin{array}{r}1.44818 \\
(0.024988)\end{array}$ & $\begin{array}{r}1.52916 \\
(0.025776)\end{array}$ \\
\hline 40 & $\begin{array}{r}1.76606 \\
(0.012763)\end{array}$ & $\begin{array}{r}1.96735 \\
(0.012456)\end{array}$ & $\begin{array}{r}2.12581 \\
(0.011955)\end{array}$ \\
\hline 60 & $\begin{array}{r}1.94527 \\
(0.008429)\end{array}$ & $\begin{array}{r}2.21469 \\
(0.008322)\end{array}$ & $\begin{array}{r}2.44627 \\
(0.008047)\end{array}$ \\
\hline 80 & $\begin{array}{r}2.07237 \\
(0.006304)\end{array}$ & $\begin{array}{r}2.36455 \\
(0.006255)\end{array}$ & $\begin{array}{r}2.62396 \\
(0.006071)\end{array}$ \\
\hline 100 & $\begin{array}{r}2.15873 \\
(0.005034)\end{array}$ & $\begin{array}{r}2.47250 \\
(0.005010) \\
\end{array}$ & $\begin{array}{r}2.75845 \\
(0.004880) \\
\end{array}$ \\
\hline $\mathrm{n}$ & \multicolumn{3}{|c|}{ Inverse Levy Prior } \\
\hline 20 & $\begin{array}{r}1.23549 \\
(0.025422)\end{array}$ & $\begin{array}{r}1.31738 \\
(0.024519)\end{array}$ & $\begin{array}{r}1.39072 \\
(0.023289)\end{array}$ \\
\hline 40 & $\begin{array}{r}1.66838 \\
(0.012605)\end{array}$ & $\begin{array}{r}1.84774 \\
(0.012314)\end{array}$ & $\begin{array}{r}1.97503 \\
(0.0117967)\end{array}$ \\
\hline 60 & $\begin{array}{r}1.87576 \\
(0.008380)\end{array}$ & $\begin{array}{r}2.10021 \\
(0.008254)\end{array}$ & $\begin{array}{r}2.30080 \\
(0.007957)\end{array}$ \\
\hline 80 & $\begin{array}{r}2.011420 \\
(0.006276)\end{array}$ & $\begin{array}{r}2.26947 \\
(0.006214)\end{array}$ & $\begin{array}{r}2.49758 \\
(0.006016)\end{array}$ \\
\hline 100 & $\begin{array}{r}2.30955 \\
(0.005017)\end{array}$ & $\begin{array}{r}2.39526 \\
(0.004983)\end{array}$ & $\begin{array}{r}2.65130 \\
(0.004843)\end{array}$ \\
\hline
\end{tabular}


SINDHU ET AL.

Table 6. The $95 \%$ credible intervals for $\tau \in 2.5$.

\begin{tabular}{|c|c|c|c|}
\hline \multirow{2}{*}{$\mathrm{n}$} & \multicolumn{3}{|c|}{ Uniform Prior } \\
\hline & Lower Limit & Upper Limit & Difference \\
\hline 20 & 2.10503 & 5.23490 & 3.12987 \\
\hline 40 & 2.44587 & 4.67921 & 2.23334 \\
\hline 60 & 2.58722 & 4.39961 & 1.81239 \\
\hline 80 & 2.71041 & 4.29493 & 1.58452 \\
\hline 100 & 2.77531 & 4.19040 & 1.41509 \\
\hline$n$ & \multicolumn{3}{|c|}{ Exponential Prior } \\
\hline 20 & 1.84980 & 4.60018 & 2.75038 \\
\hline 40 & 2.28485 & 4.37117 & 2.08632 \\
\hline 60 & 2.47071 & 4.20149 & 1.73078 \\
\hline 80 & 2.61670 & 4.14644 & 1.52974 \\
\hline 100 & 2.69796 & 4.07361 & 1.37565 \\
\hline $\mathrm{n}$ & \multicolumn{3}{|c|}{ Gamma Prior } \\
\hline 20 & 1.06688 & 2.58544 & 1.51856 \\
\hline 40 & 1.60787 & 3.04682 & 1.43895 \\
\hline 60 & 1.91272 & 3.23551 & 1.32279 \\
\hline 80 & 2.13391 & 3.36978 & 1.23587 \\
\hline 100 & 2.27978 & 3.43369 & 1.15391 \\
\hline $\mathrm{n}$ & \multicolumn{3}{|c|}{ Inverse Levy Prior } \\
\hline 20 & 0.86467 & 2.17747 & 1.31280 \\
\hline 40 & 1.41811 & 2.72520 & 1.30709 \\
\hline 60 & 1.74630 & 2.97690 & 1.23060 \\
\hline 80 & 1.98529 & 3.15093 & 1.16564 \\
\hline 100 & 2.14761 & 3.24636 & 1.09875 \\
\hline
\end{tabular}

\section{Graphical Representation of Posterior Risks under Different Priors}

The graphs reveal that posterior risks under different informative and non informative priors. It is observed that both the priors (uniform and exponential) yield the approximately the identical posterior inferences under ELF and SLELF. 


\section{LEFT CENSORED DATA FROM THE GUMBEL TYPE II DISTRIBUTION}

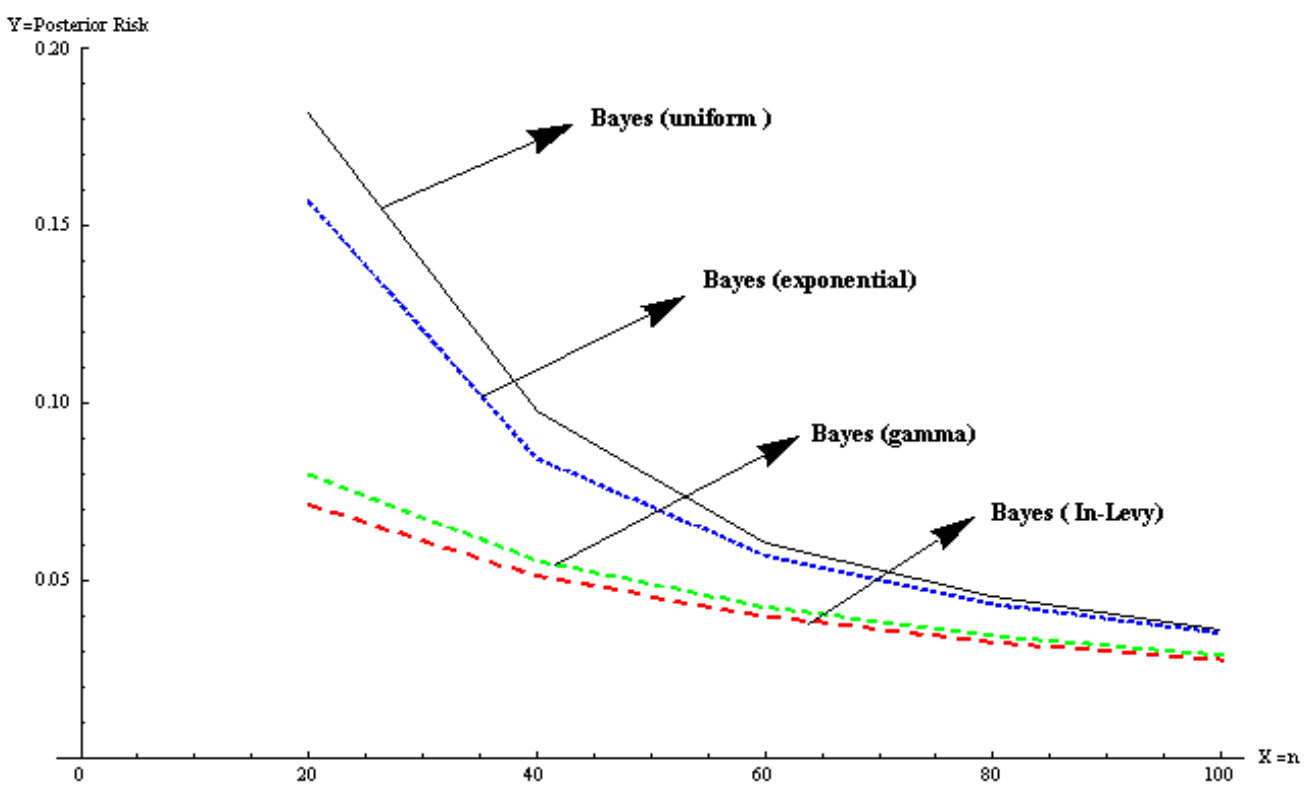

Figure 1. Effect of posterior risk under PLF with no censoring

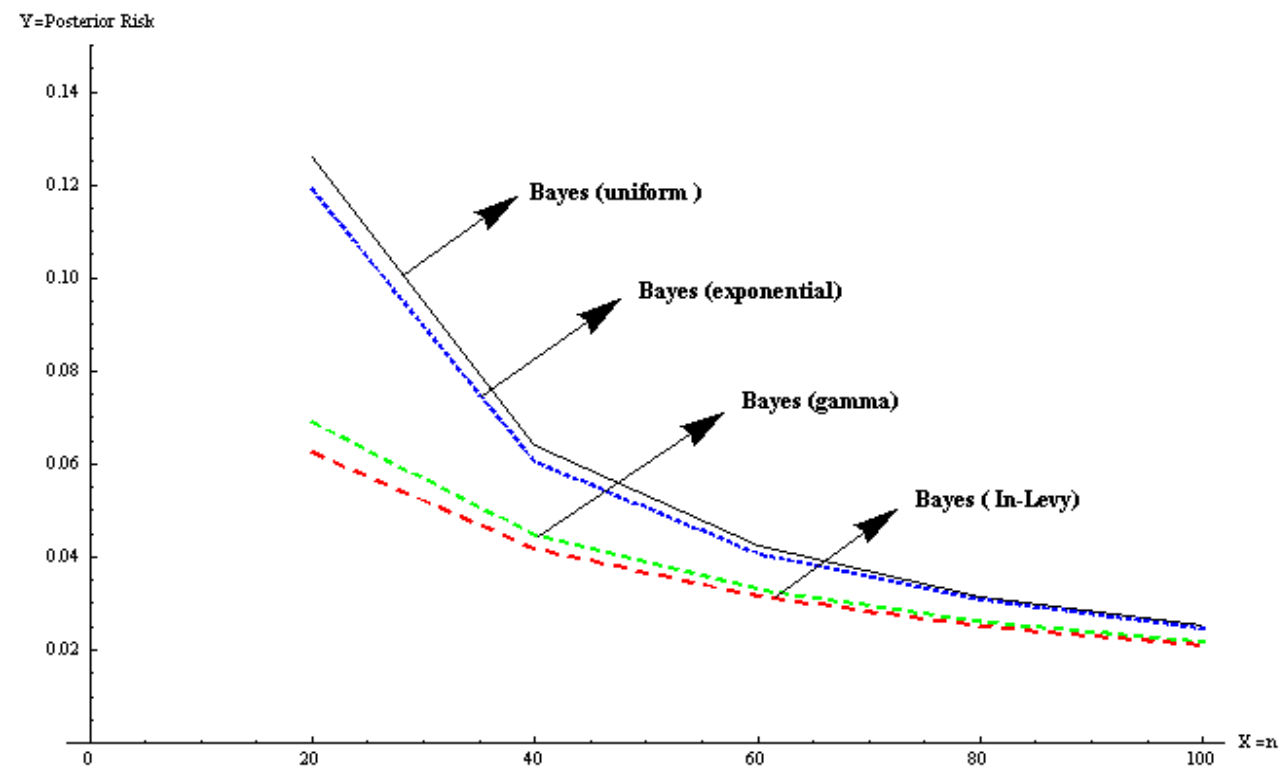

Figure 2. Effect of posterior risk under PLF with $10 \%$ censoring 


\section{SINDHU ET AL.}

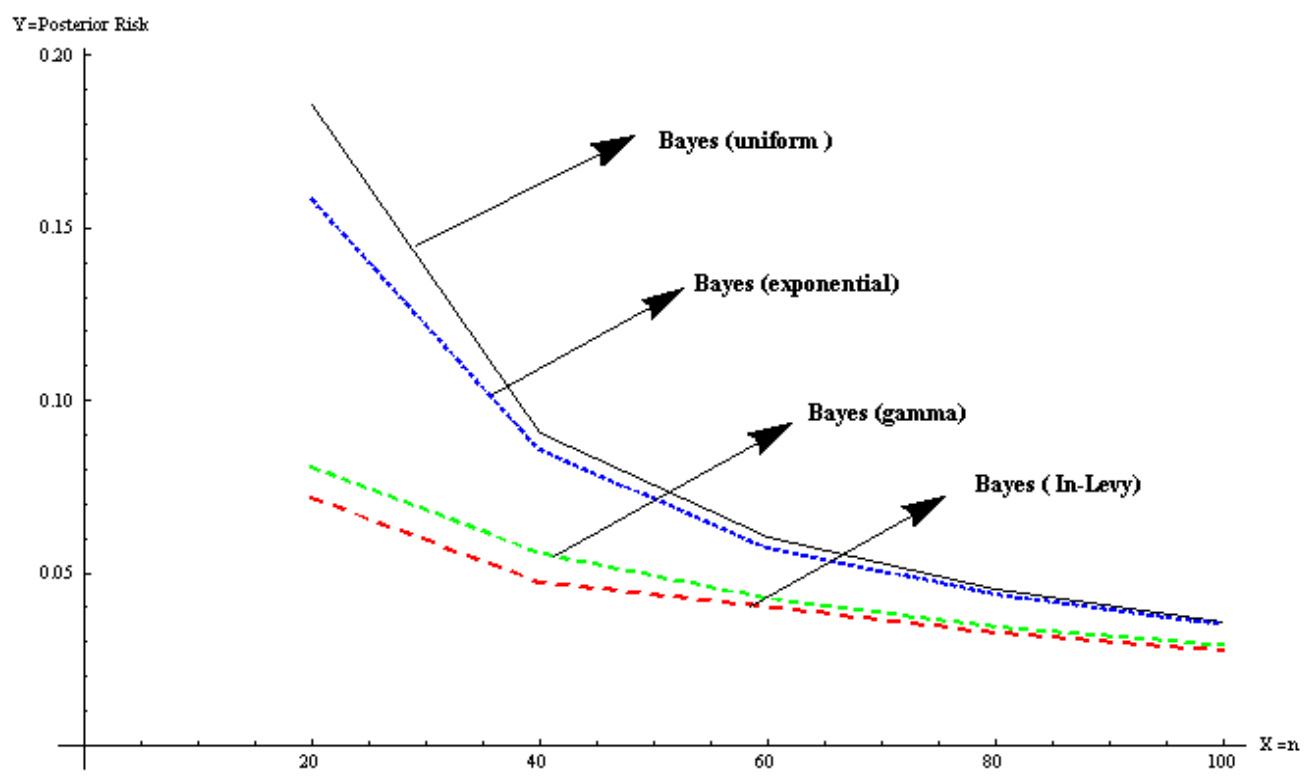

Figure 3. Effect of posterior risk under WSELF with no censoring

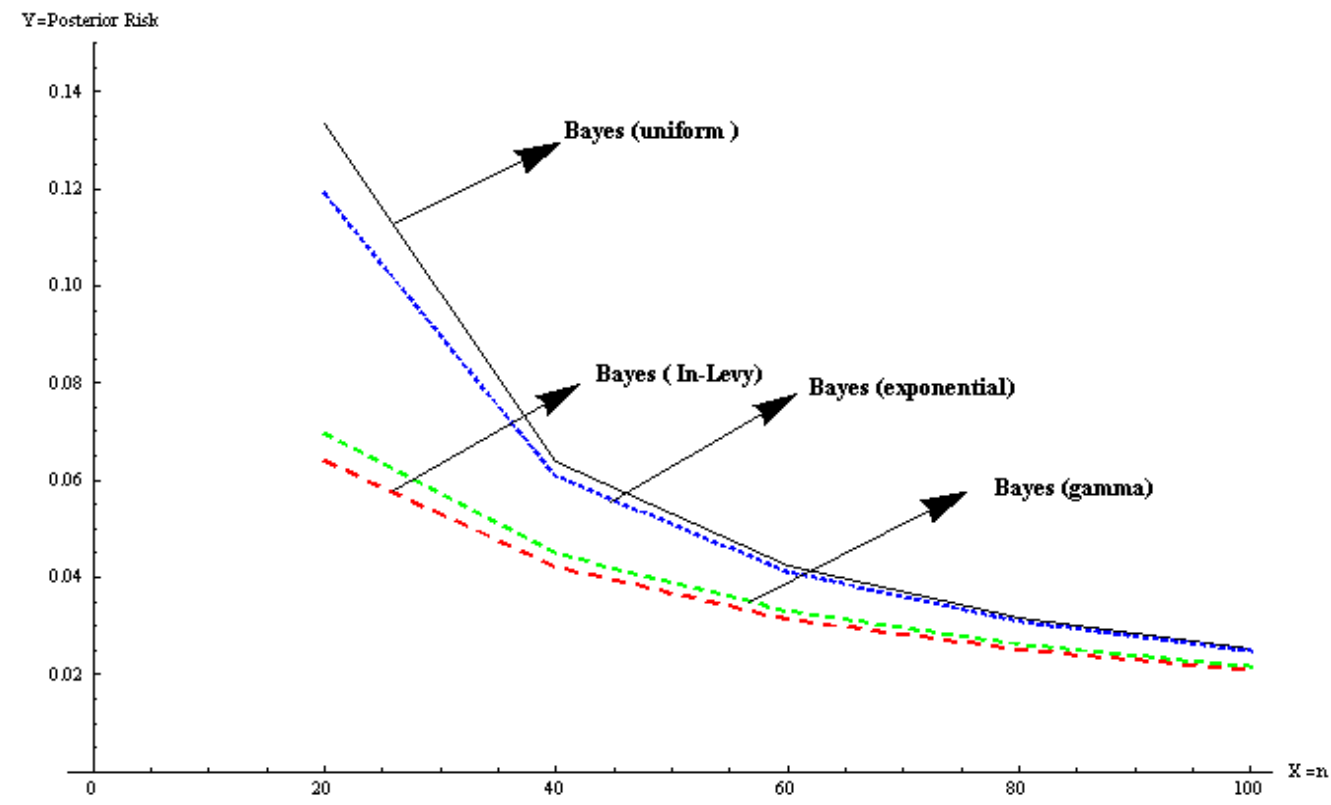

Figure 4. Effect of posterior risk under WSELF with $10 \%$ censoring 


\section{LEFT CENSORED DATA FROM THE GUMBEL TYPE II DISTRIBUTION}

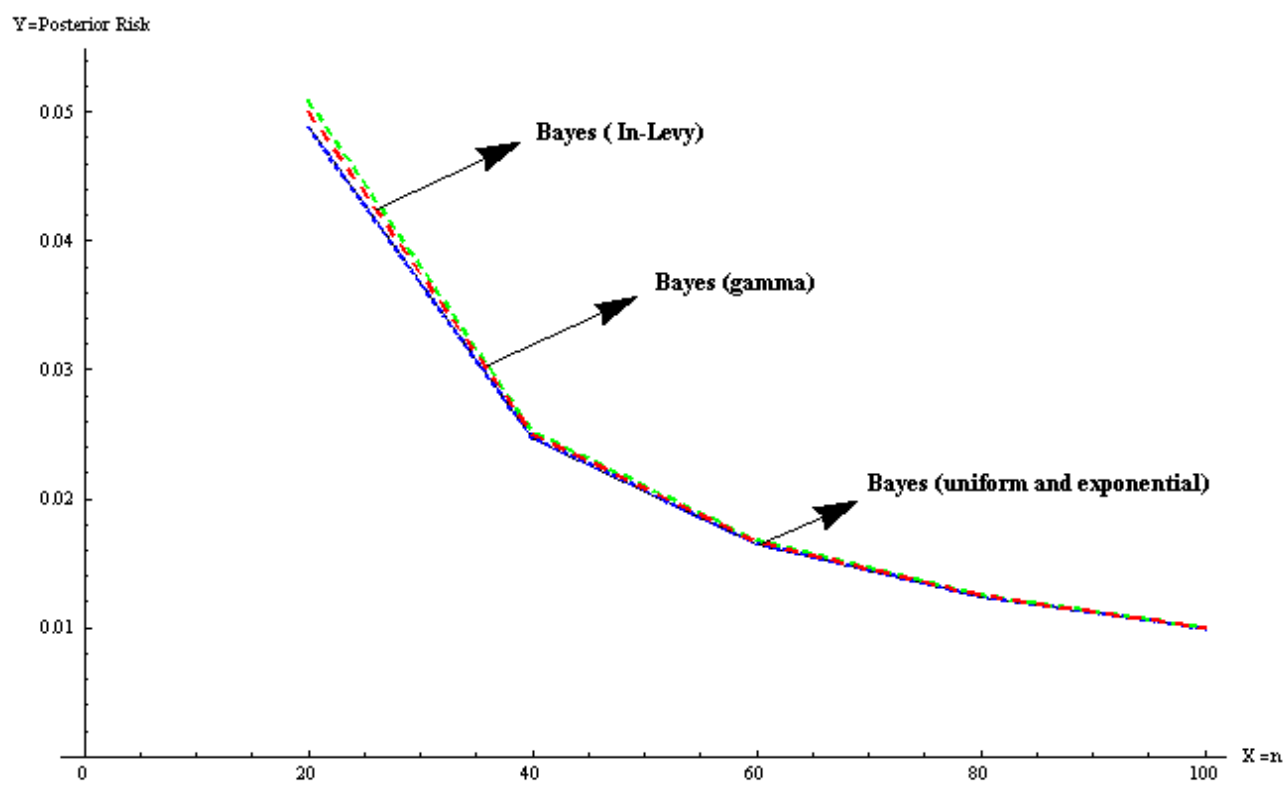

Figure 5. Effect of posterior risk under SLELF with no censoring

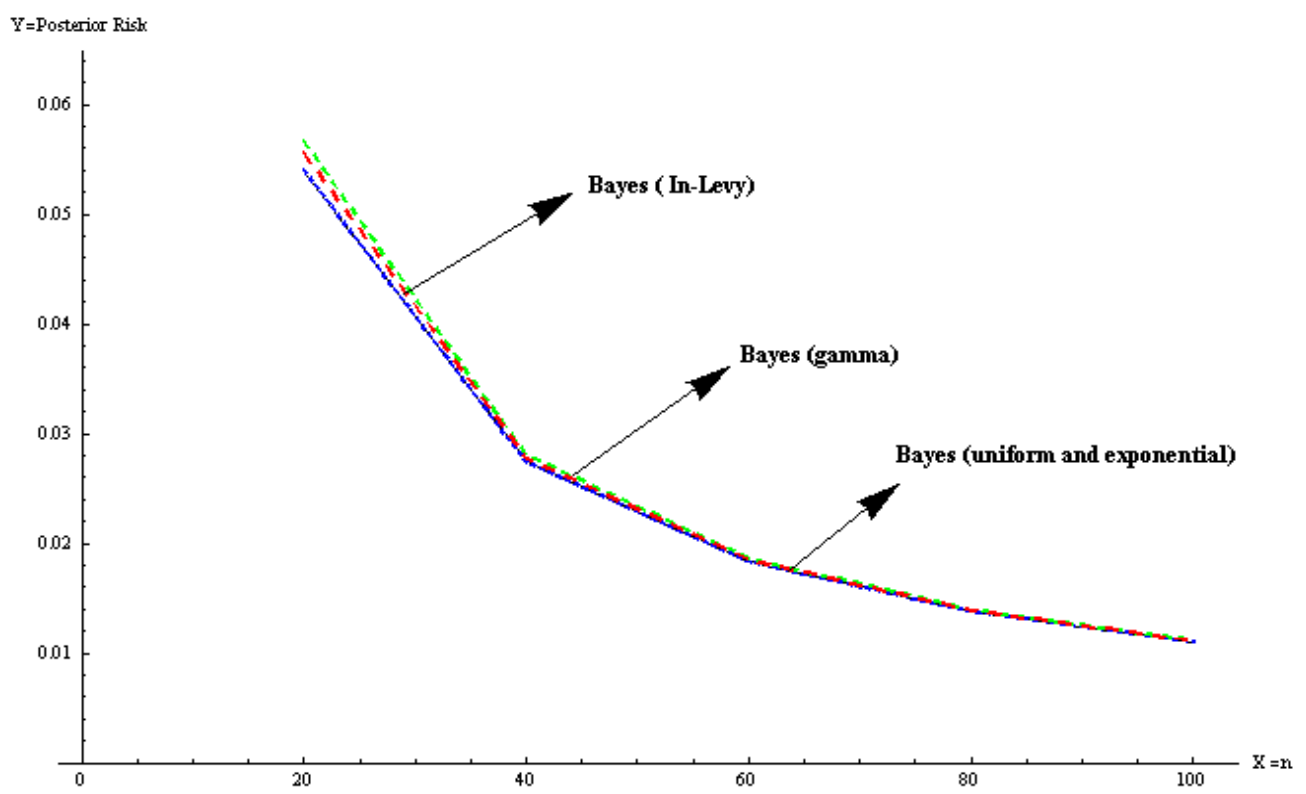

Figure 6. Effect of posterior risk under SLELF with $10 \%$ censoring 


\section{SINDHU ET AL.}

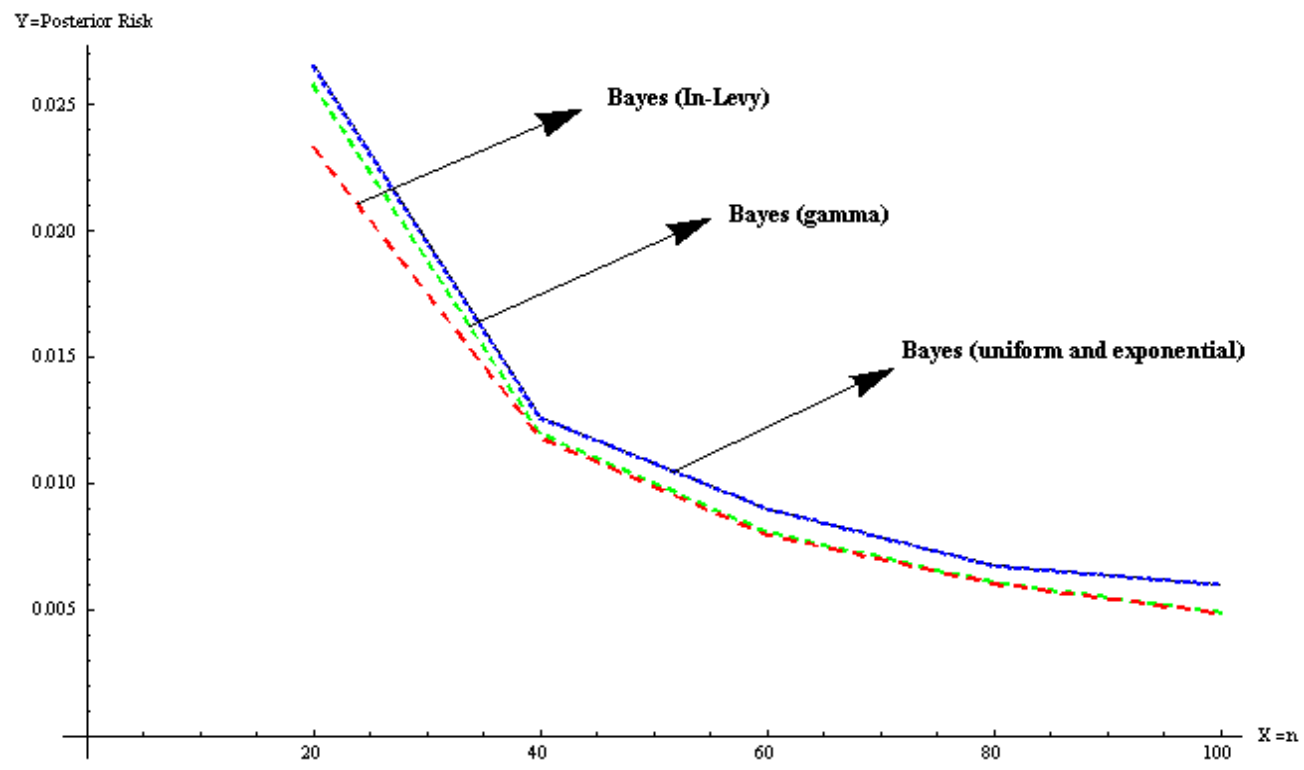

Figure 7. Effect of posterior risk under ELF with no censoring

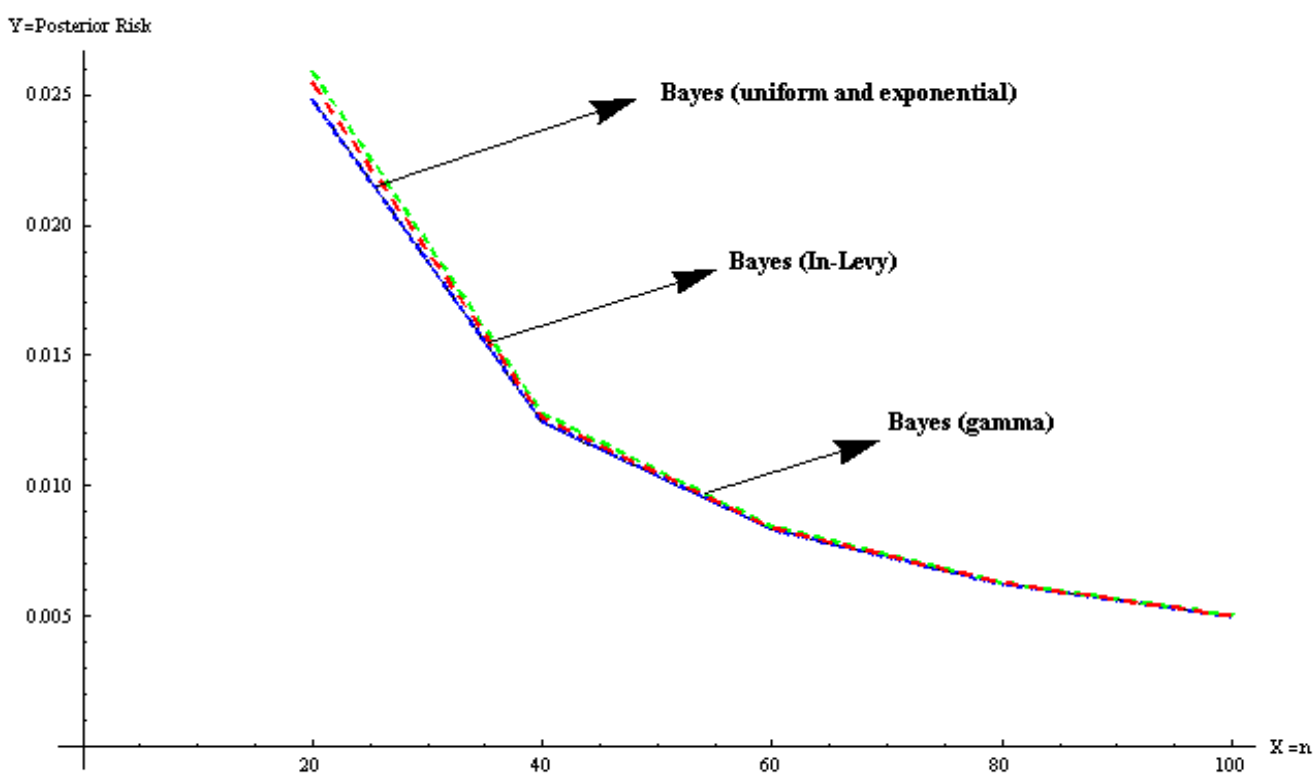

Figure 8. Effect of posterior risk under ELF with $10 \%$ censoring 


\section{LEFT CENSORED DATA FROM THE GUMBEL TYPE II DISTRIBUTION}

\section{Conclusion}

The simulation study displayed some interesting properties of the Bayes estimates. The risks under said loss functions are reduced as the sample size increases. The effect of censoring on estimation of $\tau$ is in the form of overestimation under uniform and exponential priors and underestimation assuming gamma and inverse Levy priors. Larger degrees of censoring results in bigger sizes of over or underestimation.

However, the parameter $\tau$ is either underestimated or overestimated depending upon the prior distribution to be used when censoring is not done. Then extent of this over or under estimation is directly proportional to amount of censoring rates and inversely proportional to the sample size. Further, the increase in sample size reduces the posterior risks of $\tau$.

Another interesting remark concerning the risks of the estimates is that increasing (decreasing) the censoring rate increasing (reduces) the risks of the estimates under said loss functions. The performance of squared-log error loss function and entropy loss function is independent of choice of parametric value. In comparison of informative priors and the uniform prior, the inverse Levy prior provides the better estimates as the corresponding risks are least under said loss functions except ELF and SLELF. Although the uniform and the exponential priors are equally efficient under ELF and SLELF, therefore they produce more efficient estimates as compared to the other informative priors.

The credible intervals are in accordance with the point estimates, that is, the width of credible interval is inversely proportional to sample size. From the Table 6, appended above, it can be revealed that the effect of the prior information is in the form of narrower width of interval. The credible interval assuming inverse Levy prior is much narrower than the credible intervals assuming informative and non-informative priors.

It is the use of prior information that makes a difference in terms of gain in precision. To see the effects of the posterior risks assuming different priors Figures 1-8 are prepared. It is observed from all the figures that posterior risk decreases with the increase in sample size under all loss functions. It is evident from Figures 5-8 that behavior of posterior risks is similar in all aspects. The study can further be extended by considering generalized versions of the distribution under variety of circumstances. 


\section{SINDHU ET AL.}

\section{References}

Aslam, M. (2003). An application of prior predictive distribution to elicit the prior density. Journal of Statistical Theory and Applications, 2(1), 70-83.

Bagger, J. (2005). Wage growth and turnover in Denmark. Denmark: University of Aarhus.

Bansal, A. K. (2007). Bayesian Parametric Inference. New Delhi, India: Narosa Publishing House.

Coburn, A. F., McBride, T. D., \& Ziller, E. C. (2002). Patterns of Health Insurance Coverage among Rural and Urban Children. Medical Care Research and Review, 59(3), 272-292. doi: 10.1177/1077558702059003003

Corsini, G., Gini, F., Greco, M. V., \& Verrazzani, L. (2002). Cramer-Rao bounds and estimation of the parameters of the Gumbel distribution. IEEE Transactions on Aerospace and Electronic Systems, 31(3), 1202-1204. doi: $10.1109 / 7.395217$

Grimshaw, S. D. (1993). Computing maximum likelihood estimates for the generalized Pareto distribution. Technometrics, 35(2), 185-191.

doi: 10.1080/00401706.1993.10485040

Grimshaw, S. D., Collings, B. J., Larsen, W. A., \& Hurt, C. R. (2001). Eliciting Factor Importance in a Designed Experiment. Technometrics, 43(2), 133-146. doi: 10.1198/004017001750386251

Jenkinson, D. (2005). The elicitation of probabilities: A review of the statistical literature. BEEP Working Paper. Department of Probability and Statistics, Sheffield: University of Sheffield.

Kadane, J. B. (1980). Predictive and Structural Methods for Eliciting Prior Distributions. In H. Jeffreys \& A. Zellner (Eds.), Bayesian analysis in econometrics and statistics: Essays in honor of Harold Jeffreys. Amsterdam: North-Holland.

Kotz, S., \& Nadarajah, S. (2000). Extreme value distributions. Theory and applications. London: Imperial College Press.

León, C. J., Vázquez-Polo, F. J., \& González, R. L. (2003). Elicitation of Expert Opinion in Benefit Transfer of Environmental Goods. Environmental and Resource Economics, 26(2), 199-210. doi: 10.1023/A:1026307420804

Mousa, M. A M. A., Jaheen, Z. F., \& Ahmad, A. A. (2002). Bayesian estimation, prediction and characterization for the Gumbel model based on 


\section{LEFT CENSORED DATA FROM THE GUMBEL TYPE II DISTRIBUTION}

records. Statistics: A Journal of Theoretical and Applied Statistics, 36(1), 65-74. doi: 10.1080/02331880210929

O'Hagan, A., Buck, C. E., Daneshkhah, A., Eiser, J. R., Garthwaite, P. H., Jenkinson, D. J., ... Rakow, T. (2006). Uncertain Judgements: Eliciting experts' probabilities. Chichester: John Wiley \& Sons.

Saleem, M. \& Aslam, M. (2009). On Bayesian analysis of the Rayleigh survival time assuming the random censor time. Pakistan Journal of Statistics, 25(2), 71-82. 